Tendências em Matemática Aplicada e Computacional, 3, No. 1 (2002), 141-145.

(C) Uma Publicação da Sociedade Brasileira de Matemática Aplicada e Computacional.

\title{
Higher Derivations on Lie Ideals
}

C. HAETINGER ${ }^{1}$ Departamento de Ciências Exatas e Biológicas, Centro Universitário UNIVATES, 95900-000 Lajeado, RS, Brazil, e-mail: chaet@fates.tche.br

\begin{abstract}
In this paper we present a brief proof of a recently proved result [5, Corollary 1.4]. The main result states that if $R$ is a prime ring of characteristic different of 2 and $U$ is a Lie ideal of $R$ where $U \not \subset Z(R)$, the center of $R, u^{2} \in U$ for all $u \in U$, and $D$ is a Jordan higher derivation of $U$ into $R$, then $D$ is a higher derivation of $U$ into $R$. This result extends a theorem of Awtar [1].
\end{abstract}

\section{Introduction}

Let $R$ be a ring not necessarily with an identity element. An additive subgroup $U$ of $R$ is said to be a Lie ideal of $R$ if the commutator $[u, r]=u r-r u \in U$ for every $u \in U$ and $r \in R$. It is easy to see that every bilateral ideal $A$ of $R$ is a Lie ideal of $R$.

The relationship between usual derivations and Lie ideals of prime rings has been extensively studied in the last 30 years. In particular, when this relationship involves the action of the derivations on Lie ideals. Many of these results extend other ones proven previously just for the action of the derivations on the whole ring. We have interest in investigating the action of the higher derivations on Lie ideals of prime rings.

We will consider central and noncentral Lie ideals from a different approach. There is a particular interest in Lie ideals $U$ such that $u^{2} \in U$ for every $u \in U$. Such a distinction already appears in several papers involving usual derivations and Lie ideals ([1], [2], [8]).

In 1984, Awtar [1] extended to Lie ideals a well-known result proved by Herstein concerning derivations in prime rings [7, Theorem 3.1]. In fact, Awtar proved that if $U$ is a Lie ideal of a prime ring $R$ of characteristic different of 2 such that $u^{2} \in U$ for every $u \in U$ and $d: R \rightarrow R$ is an additive mapping such that $\left.d\right|_{U}$ is a Jordan derivation of $U$ into $R$, then $\left.d\right|_{U}$ is a derivation of $U$ into $R$.

In [5, Corollary 1.4] we have generalized this result to higher derivations, by using Jordan triple higher derivations. The main purpose of this paper is to present a brief proof of the above result.

\footnotetext{
${ }^{1}$ Partially supported by Fundação de Amparo à Pesquisa do Estado do Rio Grande do Sul (FAPERGS, Brazil).
} 


\section{Definitions and Main Result}

Throughout this paper $R$ is a ring with center $Z(R)$ and $U$ is a Lie ideal of $R$. Also, $I N$ denotes the set of natural numbers including 0 , and $D=\left(d_{i}\right)_{i \in \mathbb{N}}$ is a family of additive mappings of $R$ such that $d_{0}=i d_{R}$.

In the main result of this paper we assume that the Lie ideal $U$ is not contained in $Z(R)$ and $u^{2} \in U$, for every $u \in U$. A Lie ideal of this type will be called an admissible Lie ideal. We begin with the following

Definition 2.1 If $U$ is a Lie ideal of $R$, then $D$ is said to be:

a higher derivation (HD, for short) of $U$ into $R$ if for every $n \in I N$ we have $d_{n}(a b)=\sum_{i+j=n} d_{i}(a) d_{j}(b)$, for all $a, b \in U$;

a Jordan higher derivation (JHD, for short) of $U$ into $R$ if for every $n \in I N$ we have $d_{n}\left(a^{2}\right)=\sum_{i+j=n} d_{i}(a) d_{j}(a)$, for all $a \in U$.

We prove the following:

Theorem 2.1. Let $R$ be a prime ring of characteristic different of 2 and $U$ an admissible Lie ideal of $R$. Then every JHD of $U$ into $R$ is a HD of $U$ into $R$.

\section{Prerequisites}

The following well-known result [2, Lemma 4] will be used in the paper.

Lema 3.1. Let $R$ be a prime ring of characteristic different of 2 and $U$ a Lie ideal of $R$ with $U \not \subset Z(R)$. If $a, b \in R$ and $a U b=0$, then either $a=0$ or $b=0$.

Assume that $U$ is a Lie ideal satisfying the condition $u^{2} \in U$, for every $u \in U$. For $u, v \in U,(u v+v u)=(u+v)^{2}-\left(u^{2}+v^{2}\right)$ and so $u v+v u \in U$. Also, $[u, v]=u v-v u \in U$ and it follows that $2 u v \in U$. Hence $4 u v w=2(2 u v) w \in U$, for every $u, v, w \in U$. This remark will be freely used in the whole paper.

The following lemmas can be found in [5, Lemmas 2.2 and 2.3].

Lema 3.2. Let $R$ be a 2-torsion-free semiprime ring (resp. prime ring) and $U$ an admissible Lie ideal of $R$. If $a, b \in R$ (resp. $a \in U$ and $b \in R$ ) are such that $a x b+b x a=0$, for every $x \in R$ (resp. $x \in U)$, then axb $=b x a=0$ for every $x \in R$ (resp. $a=0$ or $b=0$ ).

Lema 3.3. Assume that $R$ is a 2-torsion-free semiprime ring (resp. prime ring) and $U$ is an admissible Lie ideal of $R$. Let $G_{1}, G_{2}, \ldots, G_{n}$ be additive groups, $S: G_{1} \times \ldots \times G_{n} \rightarrow R$ and $T: G_{1} \times \ldots \times G_{n} \rightarrow R$ mappings which are additive in each argument. If $S\left(a_{1}, \ldots, a_{n}\right) x T\left(a_{1}, \ldots, a_{n}\right)=0$, for every $x \in R$ (resp. $x \in U), a_{i} \in G_{i}, i=1,2, \ldots, n$, then $S\left(a_{1}, \ldots, a_{n}\right) x T\left(b_{1}, \ldots, b_{n}\right)=0$, for every $x \in R, a_{i}, b_{i} \in G_{i}, i=1,2, \ldots, n$ (resp. $S\left(a_{1}, \ldots, a_{n}\right)=0$, for every $a_{i} \in G_{i}$, $i=1,2, \ldots, n$, or $T\left(b_{1}, \ldots, b_{n}\right)=0$, for every $\left.b_{i} \in G_{i}, i=1,2, \ldots, n\right)$. 
For elements $a, b \in R$ we put $[a, b]=a b-b a$.

The following result was proved in ([8], Lemma 7).

Lema 3.4. Let $U_{1}, U_{2}$ be Lie ideals of $R$ with $\left[U_{1}, U_{2}\right] \subset Z(R)$. Then either $U_{1} \subset Z(R)$ or $U_{2} \subset Z(R)$.

\section{Proof of Theorem 2.1}

Throughout this section $R$ is a ring, $U$ is a Lie ideal of $R$ and $D=\left(d_{i}\right)_{i \in \mathbb{N}}$ is a JHD of $U$ into $R$.

Lema 4.1. For every $u, v, w \in U$ and for $n \in \mathbb{I}$, we have:

(i) $d_{n}(u v+v u)=\sum_{i+j=n} d_{i}(u) d_{j}(v)+d_{i}(v) d_{j}(u)$;

(ii) $d_{n}(u v u)=\sum_{i+j+k=n} d_{i}(u) d_{j}(v) d_{k}(u)$;

(iii) $d_{n}(u v w+w v u)=\sum_{i+j+k=n}\left(d_{i}(u) d_{j}(v) d_{k}(w)+d_{i}(w) d_{j}(v) d_{k}(u)\right)$.

Proof. The proofs of $(i)$ and $(i i)$ are similar to the corresponding proofs in [5, Lemma 3.1].

(iii) Put $\gamma=d_{n}((u+w) v(u+w))$ and compute it by using (ii). It follows that

$$
\begin{aligned}
\gamma= & \sum_{i+j+k=n} d_{i}(u) d_{j}(v) d_{k}(u)+\sum_{i+j+k=n} d_{i}(u) d_{j}(v) d_{k}(w)+ \\
& \sum_{i+j+k=n} d_{i}(w) d_{j}(v) d_{k}(u) \sum_{i+j+k=n} d_{i}(w) d_{j}(v) d_{k}(w)
\end{aligned}
$$

Also $\gamma=d_{n}(u v u)+d_{n}(w v w)+d_{n}(u v w+w v u)=$

$$
\sum_{i+j+k=n} d_{i}(u) d_{j}(v) d_{k}(u)+\sum_{i+j+k=n} d_{i}(w) d_{j}(v) d_{k}(w)+d_{n}(u v w+w v u) .
$$

The result follows comparing both expressions.

For every JHD $D=\left(d_{i}\right)_{i \in \mathbb{N}}$ of $U$ into $R$ we put

$$
\varphi_{n}(u, v) \doteq d_{n}(u v)-\sum_{i+j=n} d_{i}(u) d_{j}(v), \text { for every } u, v \in U \text { and } n \in I N .
$$

Note that $\varphi_{n}(u, v)=0$, for every $u, v \in U$ and $n \in I N$ if and only if $D$ is a HD of $U$ into $R$.

The proof of the two next lemmas are similar as the one in [5, Lemmas 3.2, $(i)$, and 3.3].

Lema 4.2. $\varphi_{n}$ is additive in each arguments. Also, for every $u, v \in U$ and $n \in \mathbb{I}$, we have $\varphi_{n}(v, u)=-\varphi_{n}(u, v)$. 
In what follows $R$ is a prime ring of characteristic different of 2 and $U$ is a Lie ideal of $R$ with $u^{2} \in U$, for every $u \in U$. We give only a sketch of the proof of the next lemma.

Lema 4.3. Let $n \in I N$ be and assume that $u, v \in U$. If $\varphi_{m}(u, v)=0$ for every $m<n$, then $\varphi_{n}(u, v) w[u, v]+[u, v] w \varphi_{n}(u, v)=0$, for every $w \in U$.

Proof. Take $w \in U$. Then $2 w u, 2 u v, 2 v u \in U$ and also $4 u w u, 4 v w v \in U$.

Let $\gamma=4(u v w v u+v u w u v)=u(4 v w v) u+v(4 u w u) v$. Using (ii) and (iii) of Lemma 3.1 we easily obtain

$$
d_{n}(\gamma)=4 \sum_{\eta=n}\left(d_{i}(u) d_{j}(v) d_{k}(w) d_{h}(v) d_{l}(u)+d_{i}(v) d_{j}(u) d_{k}(w) d_{h}(u) d_{l}(v)\right),
$$

where $\eta=i+j+k+h+l$, and $d_{n}(\gamma)=d_{n}((2 u v) w(2 v u)+(2 v u) w(2 u v))=$

$$
4 \sum_{r+s+t=n}\left(\left(d_{r}(u v) d_{s}(w) d_{t}(v u)+d_{r}(v u) d_{s}(w) d_{t}(u v)\right) .\right.
$$

By the inductive assumption we can substitute $d_{r}(a b)$ for $\sum_{i+j=r} d_{i}(a) d_{j}(b)$ when $r<n$, for $a=u, v$ and $b=v, u$. Thus an easy computation gives

$$
\begin{gathered}
\sum_{\eta=n}\left(d_{i}(u) d_{j}(v) d_{k}(w) d_{h}(v) d_{l}(u)-\sum_{r+s+t=n}\left(d_{r}(u v) d_{s}(w) d_{t}(v u)\right)=\right. \\
-\left(\varphi_{n}(u, v) w v u+u v w \varphi_{n}(v, u)\right) .
\end{gathered}
$$

Thus comparing both expressions of $d_{n}(\gamma)$ we obtain

$$
\varphi_{n}(u, v) w v u+u v w \varphi_{n}(v, u)+\varphi_{n}(v, u) w u v+v u w \varphi_{n}(u, v)=0 .
$$

Finally, since $\varphi_{n}(v, u)=-\varphi_{n}(u, v)$, the result follows.

All is prepared for the proof of the main result of this paper.

Proof of Theorem 2.1. Let $U$ be admissible and $D$ a JHD of $U$ into $R$.

By definition, for any $u, v \in U$ we have $\varphi_{0}(u, v)=0$. Also, by ([1], Theorem), $\varphi_{1}(u, v)=0$, for every $u, v \in U$. We proceed by induction.

Assume that $\varphi_{m}(u, v)=0$, i.e., $d_{m}(u v)=\sum_{i+j=m} d_{i}(u) d_{j}(v)$, for all $u, v \in U$ and $m<n$. Take $u, v \in U$. By Lemma 4.3 we have $\varphi_{n}(u, v) w[u, v]+[u, v] w \varphi_{n}(u, v)=0$, for every $u, v, w \in U$. It follows from Lemma 3.2 that $\varphi_{n}(u, v) w[u, v]=0$, for all $u, v, w \in U$. Thus Lemma 3.3 implies that $\varphi_{n}\left(u_{1}, v_{1}\right) w\left[u_{2}, v_{2}\right]=0$, for every $w, u_{i}, v_{i} \in U, i=1,2$. The proof can easily be completed using Lemma 3.1 and Lemma 3.4.

Remark. The result proved in [1] also holds for Lie ideals contained in the center of $R$. Actually, in this case the theorem is trivial. We were unable to answer the question on whether the corresponding result is also true for higher derivations. 
Resumo. Neste artigo apresentamos uma prova direta e mais curta de um resultado que recentemente provamos ([5], Corollary 1.4). O teorema principal estabelece que se $R$ é um anel primo de característica diferente de 2 e $U$ é um ideal de Lie de $R$ tal que $U \not \subset Z(R)$, o centro de $R, u^{2} \in U$ para todo $u \in U$, e $D$ é uma derivação de Jordan de ordem superior de $U$ em $R$, então $D$ é uma derivação de $U$ em $R$. Este resultado estende um teorema de Awtar [1].

\section{References}

[1] R. Awtar, Lie ideals and Jordan derivations of prime rings, Proc. Amer. Math. Soc., 90, No. 1 (1984), 9-14.

[2] J. Bergen, I. N . Herstein and J. W. Kerr, Lie ideals and derivations of prime rings, J. Algebra, 71 (1981), 259-267.

[3] D. R. Farkas, C. Geiss and E. N. Marcos, Smooth automorphism group schemes, in "Conference on Representations of Algebras", São Paulo, Brazil, to appear.

[4] M. Ferrero and C. Haetinger, Higher derivations of semiprime rings, Comm. Algebra, to appear.

[5] M. Ferrero and C. Haetinger, Higher derivations and a theorem by Herstein, Quaestiones Mathematicae, to appear.

[6] C. Haetinger, "Derivações de Ordem Superior em Anéis Primos e Semiprimos", Ph.D. thesis, IMUFRGS, UFRGS, Porto Alegre, RS, Brazil, 2000.

[7] I. N. Herstein, Jordan derivations of prime rings, Proc. Amer. Math. Soc., 8 (1957), 1104-1110.

[8] C. Lanski and S. Montgomery, Lie structure of prime rings of characteristic 2, Pacific J. Math., 42, No. 1 (1972), 117-136. 
